\title{
Severe Osteoporosis as the Presentation of Concealed Swyer Syndrome (Pure Gonadal Dysgenesis)
}

\author{
J A O'Hare ${ }^{1}$, K Hickey². \\ ${ }^{1}$ Department of Endocrinology and ${ }^{2}$ Department of Gynecology , University Hospital Limerick, \\ University of Limerick , Graduate Entry Medical School, Limerick, Ireland

\section{Introduction}

Swyer syndrome (SS) is a rare form of hypogonadism with male karyotype but female phenotype.

They have normal female appearance, external genitalia, vagina, tubes but streak gonads.

Are profoundly oestrogen deficient and if untreated, tall.

They have female sexual orientation.

SS is due to a mutation of the sex determining region of the Y chromosome (SRY)

This results in failure of testosterone production in-utero with nondevelopment of male genitalia. Anti-Mullerian hormone failure results in non- regression of the Mullerian duct.

\section{History and Findings}

- A 28 year presented with acute back pain

- Height $180 \mathrm{~cm}$ weight $63 \mathrm{Kg}$

- Breasts underdeveloped, normal female external genitalia.

- $\mathrm{X}$ ray: Fracture Lumbar vertebra $\mathrm{L} 2$

- DEXA Scan T score: Lumbar -4.2, Hip -2.4

- LH, FSH Oestrogen, progesterone: low Claims was menstruating since age 14

- Testosterone: low normal

- Calcium, phosphate, Parathyroid hormone and Vit D; normal.

\section{Further Investigation and a Revelation}

- Chromosome analysis 46 X Y normal male

- PCR confirms presence of SRY locus

- Anti- Mullerian factor low.

- Patient originally reported menstruating from 14 years

- Now admits always had amenorrhea.

- Ethnic group culture influenced concealment.

\section{Management 1}

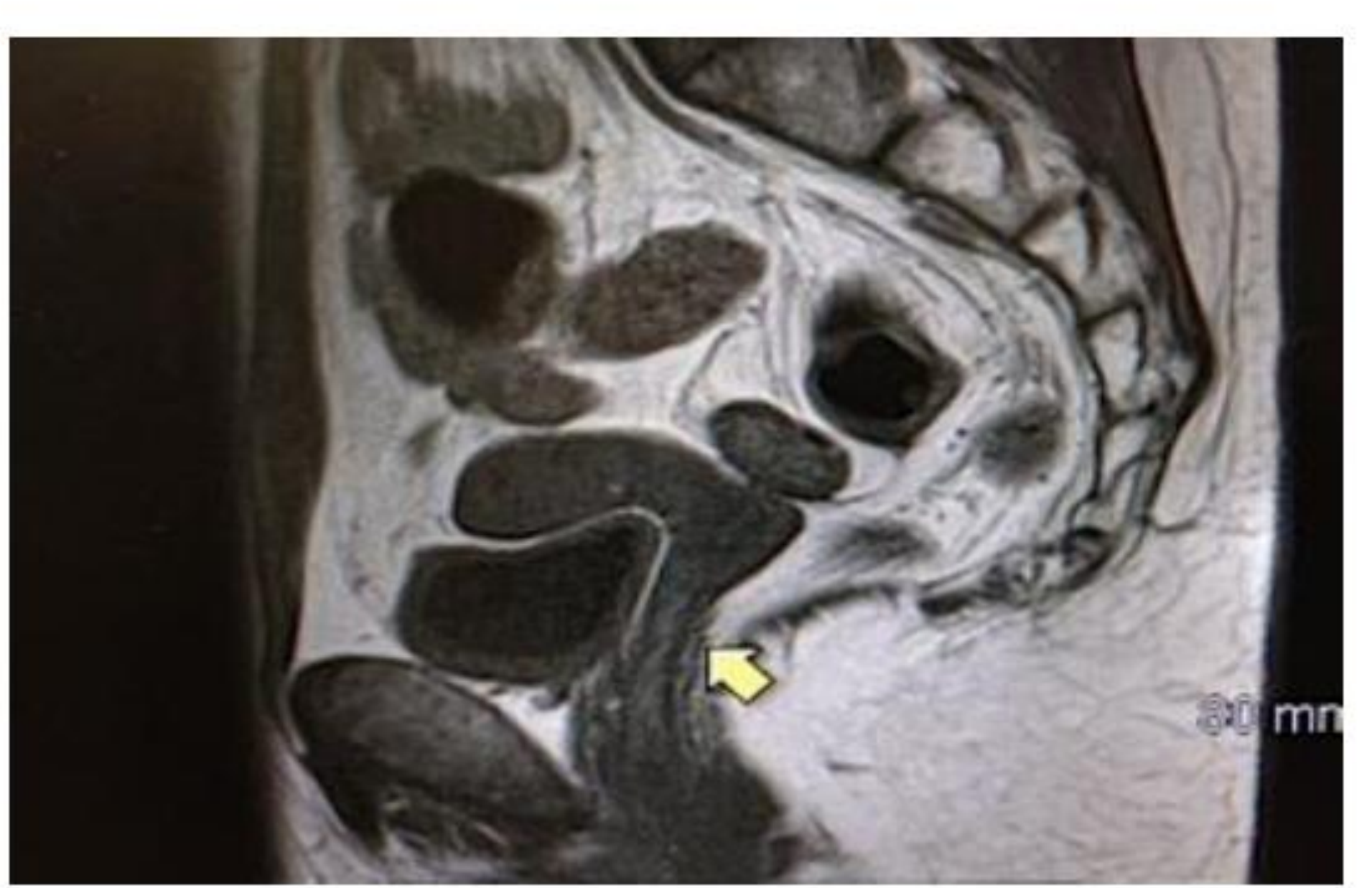

- MRI Pelvis confirms vagina, tubes, streak gonads only.

- Progesterone induced withdrawal bleed

- Cyclical oestrogen induced cyclical menses

- Breasts further developed

- Height increases despite oestrogen to $184 \mathrm{cms}$

- Patient counselled

- Laparoscopic gonadectomy- no malignancy

- Advised re possibility of donor ovum /implantation

Management 2: Osteoporosis

DEXA Scan Base Line line and +24 months
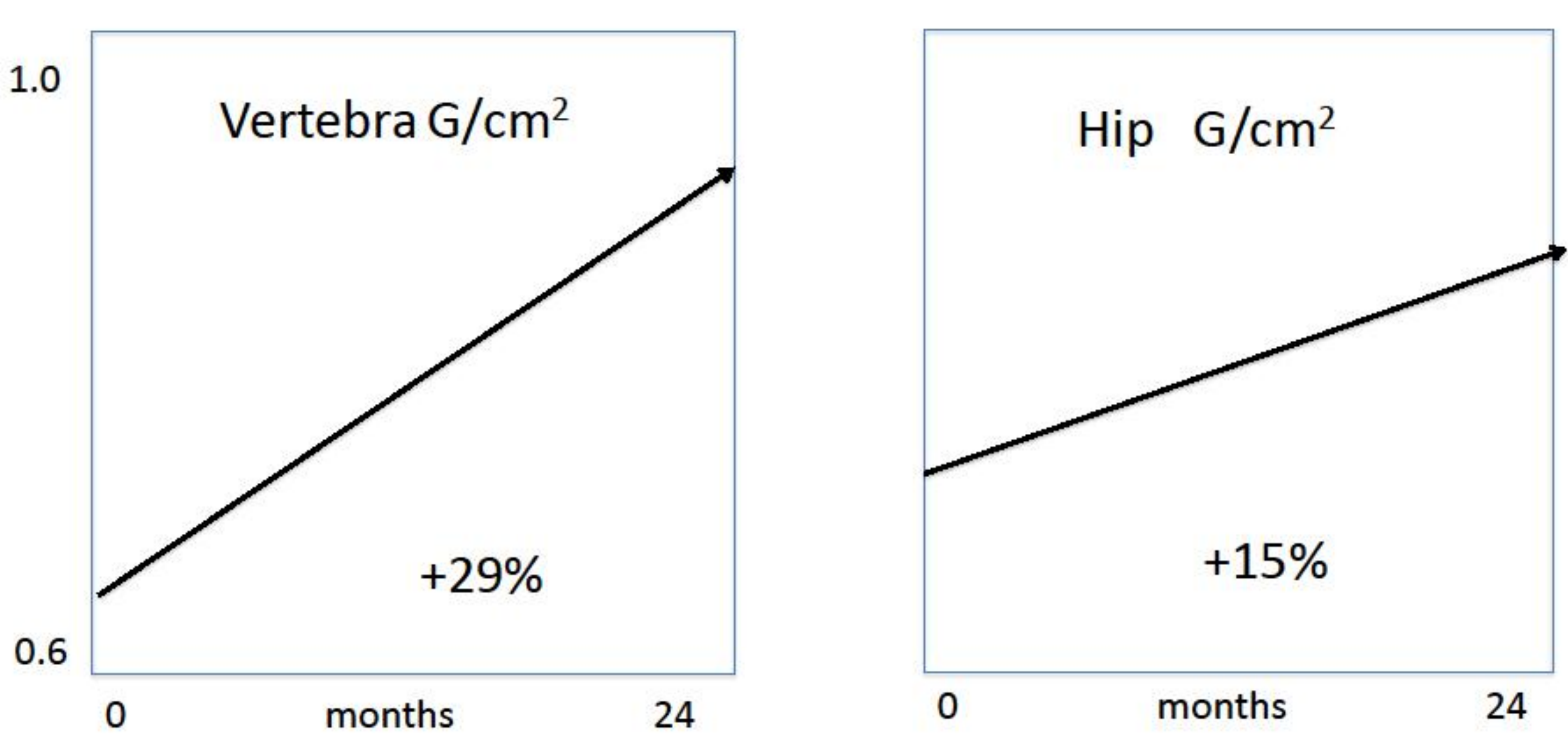

Teriparatide s.c. for 2 years + vit D + calcium + oestrogen

\section{Summary}

- Late presentation of Swyer Syndrome resulted in preventable severe osteoporosis,

And unwanted height,

- Due to concealment of amenorrhea for 14 years for cultural reasons 